\title{
Acute Dose of Intranasal Oxytocin Improves Recognition of Happy Faces in Musicians with High Levels of Music Performance Anxiety
}

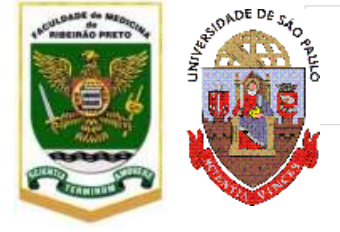

\section{INTRODUCTION}

- Music performance anxiety (MPA) is characterized by persistent and intense apprehension in situations of public musical performance that is disproportional to the level of aptitude, practice, and preparation of the musician;

- Individuals with MPA present physical, behavioral, and cognitive manifestations of anxiety, in addition to information processing deficits, especially in facial emotion recognition (FER);

- Recentes studies have provided evidence for the therapeutic potential of oxytocin (OCT), a neuropeptide produced in the hypothalamus of the brain, connected to its anxiolytic properties and action upon different cognitive processes including confidence, FER, cooperation, and social dysfunction

\section{OBJECTIVE}

To assess the effects of an acute dose of oxytocin (24IU) on the recognition of facial expressions of emotion (RFEE) in musicians with music performance anxiety (MPA).

\section{METHODS}

- Cross-over, clinical, randomised, double-blinded, placebocontrolled trial

- Inclusion: age above 18 years; being male; professional or amateur musical practice; and frequent public performances (at least twice a month) to expressive audiences.

- 41 male musicians, were assessed in respect to their level of MPA with the Kenny Music Performance Anxiety Inventory (KMPAI): with scores $\geq-15$ are classified as having high MPA, while scores below this threshold indicate low MPA.

- Participants were randomized to receive a single nasal dose of OCT (24 IU) or placebo. Both substances were packed in identical flasks and numbered in sequence according to the randomization list. Analyses performed with ANOVA 2.0 for cross-over models.

- Facial Emotion Recognition Task "Emotion 96": computerized task with static stimuli consisting of 96 color pictures showing facial expressions of emotion (happiness, sadness, fear, anger, disgust, and emotionally neutral faces).

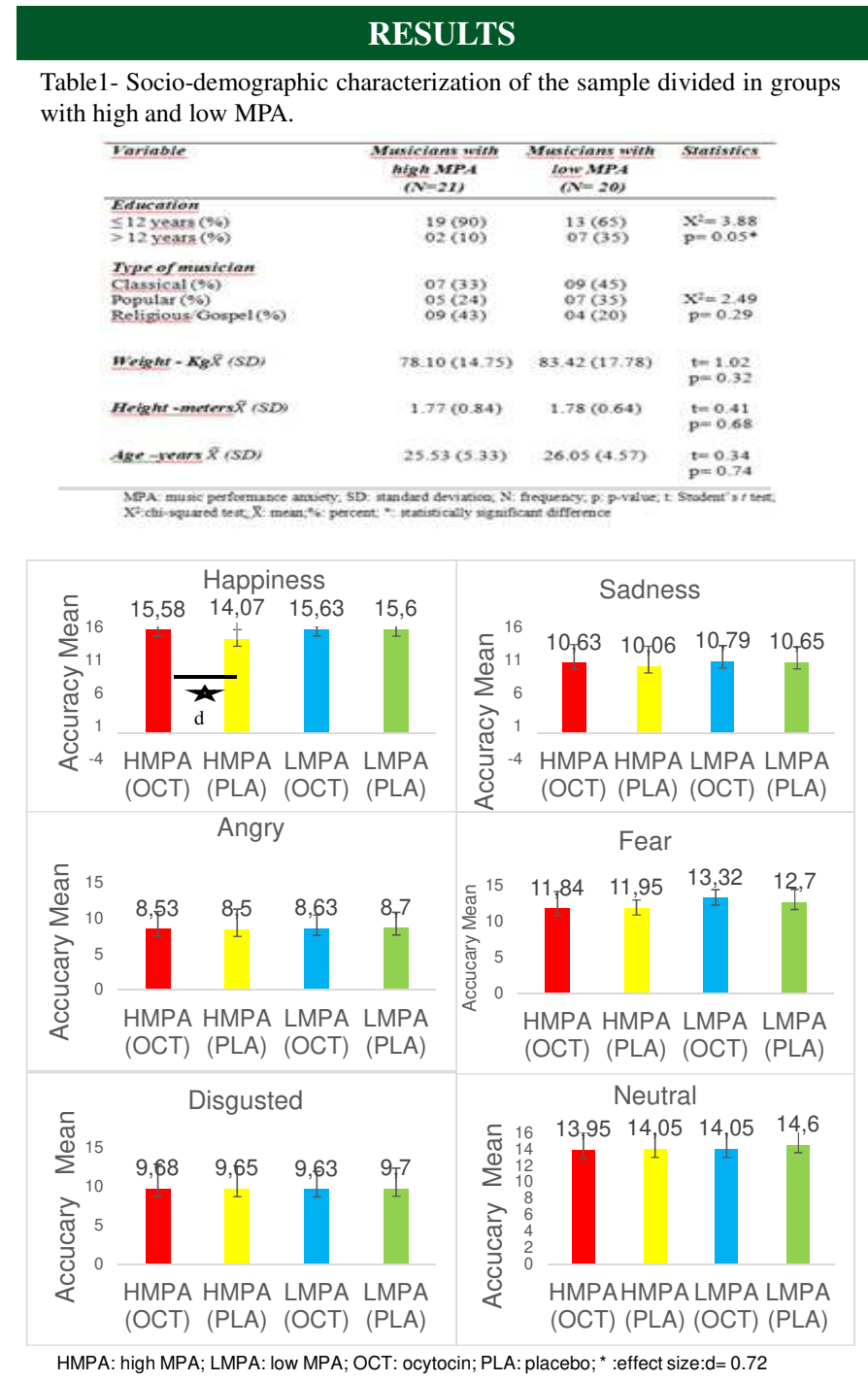

Figure 1 - Effects of oxytocin on the facial emotion recognition task "Emotion $96 "$

\section{CONCLUSION}

Oxytocin has been shown to be effective in improving the social cognition deficits in musicians with high levels of MPA, thus enabling the correct identification of clues suggesting social approval. These findings may have clinical implications as oxytocin seems to be promising in the treatment of MPA, a very prevalent condition among musicians which leads to major emotional suffering and damage to their professional career.

\section{REFERENCES}

*Salmon PG. A psychological perspective on musical performance anxiety: a review of the literature. Med Probl Perform Art, 1995.

*Arrais KC et al. Social anxiety disorder women easily recognize fearfull, sad and happy faces: the influence of gender. J Psych Res 2010. 